Théologiques

Théologiques

\title{
Foi et vision mystique dans les Confessions d'Augustin
}

\section{Jean Grondin}

Volume 16, numéro 2, 2008

L'individualisation de la relation religieuse

URI : https://id.erudit.org/iderudit/001630ar

DOI : https://doi.org/10.7202/001630ar

Aller au sommaire du numéro

\section{Éditeur(s)}

Faculté de théologie et de sciences des religions, Université de Montréal

\section{ISSN}

1188-7109 (imprimé)

1492-1413 (numérique)

Découvrir la revue

\section{Citer cet article}

Grondin, J. (2008). Foi et vision mystique dans les Confessions d'Augustin. Théologiques, 16(2), 15-30. https://doi.org/10.7202/001630ar

\section{Résumé de l'article}

La figure d'Augustin est certainement exemplaire pour étudier ce dont il y va dans la relation et l'expérience religieuses. Comme chacun sait, Augustin décrit la relation religieuse comme un long processus de conversion à Dieu, dans ce qui correspond bel et bien à un acte de foi, mais cette conversion ne va pas sans certaines expériences d'extase qu'Augustin dépeint en s'inspirant de textes de Plotin. Nous nous demanderons si les deux expériences, celles de la foi et de la vision, sont compatibles, chez Augustin plus particulièrement, mais aussi sur un plan plus général. 


\title{
Foi et vision mystique dans les Confessions d'Augustin
}

\author{
Jean GRONDIN* \\ Département de philosophie \\ Université de Montréal
}

La question que j'aimerais soulever ici est celle de savoir dans quelle mesure la foi et la vision mystique sont conciliables en général et chez Augustin en particulier. C'est que celui qui a fait une expérience mystique a nécessairement quelque chose de plus que la simple foi: il a vu ou touché une "autre réalité ». Et lorsque l'on dit que l'on a la foi, c'est généralement pour signifier que l'on n'a pas soi-même d'expérience directe de la réalité divine, mais que l'on y croit «quand même".

Cette distinction entre la foi et la vision est pointée par saint Paul lorsqu'il oppose, dans un texte célèbre (1 Co 13,12), la «vision dans un

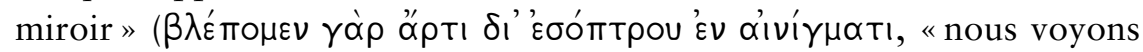
jusqu'à maintenant à travers un miroir, en énigme») à la "vision face à

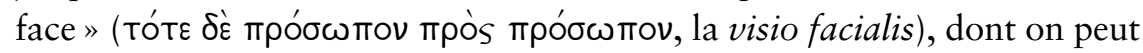
dire qu'elle s'apparente à l'expérience mystique directe. Seulement, la différence entre les deux types de vision recoupe chez saint Paul celle de notre vie terrestre et de notre existence future : tant que nous vivrons, nous n'aurons que la vision en énigme, c'est-à-dire la foi, mais après notre mort, nous bénéficierons enfin, si Dieu le veut bien, de la vision face à face. Nous

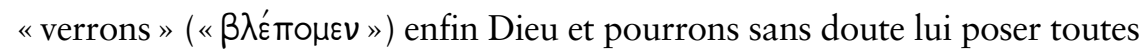
les questions que nous brûlons de lui adresser. C'est aussi de cette manière, je crois, que l'imaginaire populaire se représente la foi, c'està-dire comme une croyance en l'absence de vision, et ce que pourrait être l'existence après la mort, où nous verrons Dieu face à face. La seule, quoique importante différence est que saint Paul conçoit la relation de foi

Jean Grondin est professeur titulaire au Département de philosophie de l'Université de Montréal. Parmi ses publications, notons: L’herméneutique, Paris, PUF (Que saisje ? 3758), 2006, ainsi qu'une Introduction à la métaphysique, Montréal, PUM, 2004. 


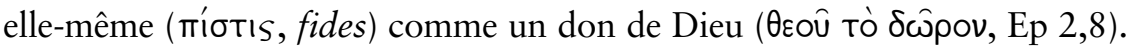
C'est une manière de dire que la "foi » est déjà le témoignage d'une présence directe, "mystique » si l'on peut dire, du divin. Avoir la foi, c'est déjà pour saint Paul jouir d'une grâce divine (il n'est que de penser ici à la manière dont saint Paul dit avoir été foudroyé sur le chemin de Damas), qui ne ressortit peut-être pas ou pas encore à la vision face à face, mais qui s'apparente néanmoins à une vision, car saint Paul dit bien qu'il s'agit d'une "vision dans un miroir", celui de la foi.

Dans le présent travail, je me propose d'étudier cette relation entre la foi et la vision telle qu'elle s'orchestre chez Augustin. C'est que si Augustin part de la foi, il n'est pas sans évoquer des expériences d'extase au cours de son existence terrestre, et même avant sa conversion pleine et entière. Il va donc plus loin que saint Paul, en disant que cette expérience est possible, voire naturelle pour l'âme qui apprend "à rentrer en elle-même». Mais cette expérience est-elle vraiment possible pour «l'œil » de l'âme humaine et aussi naturelle que semble le prétendre Augustin ?

Dans ce qui suit, j'utiliserai le terme de «mystique» ou d'extase pour caractériser l'expérience directe, voire charnelle du divin ( "vu » ou «entendu » par nos sens, y compris ceux de l'âme). C'est un usage qui diffère de celui d'Augustin, qui connaît le terme de mystica, mais qui ne l'emploie que pour désigner des choses mystérieuses qui ont besoin d'être éclaircies par le biais d'une interprétation spirituelle. C'est en ce sens que le dernier livre des Confessions parle des voces mysticas ( "paroles mystérieuses ") des Écritures saintes ${ }^{1}$, dont le propos est voilé dans des mystères (rem velatam in mysteriis, $3,5,9)$ qui ne sont pas accessibles aux superbes, mais dont le sens grandit avec les humbles. Au livre 6 des Confessions, Augustin dira, dans le même esprit, que l'exégèse pratiquée par Ambroise «soulevait le voile mystique» de l'Écriture (remoto mystico velamento) en

1. Confessions 13,21,30, voces mysticas. Voir aussi Conf. 6,4,6 (où il est question de l'exégèse pratiquée par Ambroise): remoto mystico velamento. Voir le commentaire de O'Donnell (1992) sur ce passage: "For A. the word [mystica] regularly speaks of texts or events that demand interpretation and are resolved only by a spiritual interpretation (e.g., en. Ps. 146.13, "scripturas mysticis quibusdam rebus salubriter tegentes intellegentiam"), but the link to cult activity in the word's pre-Christian usage is not broken (e.g., trin. 3.4.10, "prece mystica" [of eucharistic consecration] and trin. 15.26.46, "mystica et invisibili unctione" [of Jesus' receiving the spirit in his own baptism by John]). In Scripture only at Is. 3.3, speaking approvingly of one who is "prudens eloquii mystici"; in Christian Latin from Lactantius on. » Voir aussi De civitate Dei 11,23 : altitudo mystica. Sur l'influence d'Augustin sur la mystique chrétienne, voir Wright (1999). 
découvrant un sens spirituel là où la lettre semblait enseigner une erreur (Conf. 6,4,6; Labriolle, 123). Est donc mystique pour Augustin tout ce qui est mystérieux, d'origine divine et en attente d'explication. Le "mystique » reste pour nous mystérieux, mais renvoie plus directement à une expérience «tactile» du divin, mais qu'Augustin ne nomme jamais «mystique».

\section{L'exorde des Confessions: la foi certes, mais la recherche et l'invocation d'une présence}

Il est banal de le dire, mais les Confessions attestent d'abord la "réelle présence» de Dieu, du fait qu'elles lui sont directement adressées. Cela tombe sous le sens dès les premières lignes de ce grand texte: Magnus es, domine, et laudabilis valde $(1,1,1)$, " tu es grand, Seigneur, bien digne de louanges ». La confession d'Augustin est donc un discours que Dieu peut entendre. Or ce Dieu, il s'agit d'abord de le louer et celui qui cherche à le louer n'est qu'une infime portion de sa création, un homme dont le cœur est inquiet et qui le restera jusqu'à ce qu'il puisse reposer en Dieu, inquietum est cor nostrum, donec requiescat in te $(1,1,1)$.

Mais comment louer Dieu si on ne le connaît pas? C'est la première "aporie " des Confessions: qui peut t'invoquer s'il ne te connaît, sed quis te invocat nesciens te $(1,1,1)$ ? C'est à ce moment précis qu'intervient la toute première mention de la foi dans les Confessions: comment invoquer celui en qui on ne croit pas (quomodo autem invocabunt in quem non crediderunt) ? L'invocation présuppose ici la foi (credere), laquelle apparaît donc première dans le rapport à Dieu ${ }^{2}$. La foi présuppose, à son tour, un prédicateur qui l'a transmise: quomodo credent sine predicante? Cette primauté de la foi transmise, Augustin la souligne aussi en «citant» le Psaume 18,14: credo, propter quod et loquor, " je crois, et c'est aussi pourquoi je parle» $(1,5,6)$.

Seulement, ce discours qui procède de la foi conduit chez Augustin à une invocation, terme que l'auteur des Confessions entend littéralement,

2. Foi qui possède un sens multiple chez Augustin, car il peut s'agit d'un credere Deum, d'un credere Deo et d'un credere in Deum (voir TeSelle 1999): 1) Credere Deum ( «croire Dieu »), avec l'accusatif, c'est d'abord croire en l'existence de Dieu; 2) quant au credere Deo, il s'agit d'une croyance accordée à l'autorité des témoignages humains sur Dieu; 3) le credere in Deum désigne, pour sa part, l'adhésion plus personnelle à Dieu ou le mouvement de la foi vers Dieu. TeSelle range la division entre le contenu de la foi (ea qua creduntur) et l'acte de foi personnel (la fides qua creditur, qui peut être variable et erronée) au sein de la première acception, le credere Deum. 
comme un "vocare in se » : invoquer, c'est appeler à venir en soi. Dieu doit devenir une présence réelle en moi. Mais comment peut-il le devenir ? se demande aussitôt Augustin $(1,2,2)$. En quelle partie de moi Dieu devrait-il alors venir (et quis locus est in me, quo veniat in me deus meus?) et est-ce alors une partie de lui qui doit venir en moi ou Dieu en totalité ?

La difficulté de ces formulations est qu'elles se font une représentation spatiale de Dieu, qui devrait venir «de quelque part », en " une partie » de moi, et n'offrant peut-être qu'une partie de lui-même. Mais Augustin restera jusqu'à la fin fasciné par ces représentations un peu spatiales de Dieu (même si le platonisme, avec sa conception immatérielle de la divinité, est censé l'avoir aidé à les dépasser). Dieu doit pouvoir devenir une présence réelle en moi, doit pouvoir être "touché ». On peut dire que c'est l'amorce de l'expérience mystique dans les Confessions, qui jaillissent certes d'un acte de foi, mais qui aspirent à "toucher » Dieu, dans une expérience qui confine à la mystique. Autrement dit, si la foi — et la foi reçue d'un prédicateur (sa mère, Ambroise, ses confrères de conversion, etc.) - représente le point de départ de l'invocation, celle-ci s'oriente d'entrée de jeu sur une présence du divin, que l'acte de foi n'exclut aucunement. Bien au contraire.

Et lorsque Augustin s'adresse à Dieu au début de ses Confessions, ce qu'il lui demande avant tout, c'est de lui répondre afin qu'il puisse l'entendre: dic mibi per miserationes tuas, domine deus meus, quid sis mihi. Dic animce mece: salus tua ego sum. Sic dic, ut audiam (1,5,5: "dites-moi dans votre miséricorde, Seigneur mon Dieu, ce que vous êtes pour moi; dites à mon âme: c'est moi qui suis ton salut [Ps 34,3]. Dites cela, que je l'entende »). «Donne-moi de savoir, dis-moi » (da mibi, dic mihi) sont des impératifs qu'Augustin ne cesse d'adresser à Dieu en attendant de lui une réponse $^{3}$. Ce qu'Augustin recherche, c'est toujours aussi le visage même de Dieu, selon le Ps 41,3 (1,18,28: quœesivi vultum tuum; vultum tuum, domine, requiram). La foi est aspiration à voir le visage de Dieu et cette aspiration doit pouvoir être satisfaite.

Si l'origine des Confessions réside ainsi dans un acte de foi, qui «invoque» une réponse du créateur, la «réponse» de Dieu, si l'on peut dire, se fera entendre par le biais des textes et des voix que Dieu sèmera sur le chemin d'Augustin lors des étapes décisives de sa conversion. Il est significatif d'observer que chacun de ces moments conduira à une

3. Sans doute cette réponse viendra-t-elle par l'Écriture, mais il demeure qu'en elle, ou sous son "voile mystique », c'est toujours Dieu qui m'interpelle directement. La relation « religieuse » est ainsi une relation à une parole divine, qui n'est pas seulement pour Augustin le «témoignage de foi » des apôtres qui ont rédigé les Saintes Écritures. 
expérience «mystique» ou «extatique », comme si l'expérience d'une présence réelle devait servir de pendant à l'acte de foi lui-même. Il y a trois moments qui m'intéresseront plus particulièrement ici, celui de la découverte des livres des platoniciens (livre 7), celui de la voix entendue dans le jardin de Milan (livre 8) et celui des expériences d'extase d'Ostie (livre 9).

\section{Les libri platonicorum et les extases «imparfaites» qu'ils rendent possibles}

La découverte des livres des platoniciens aura bien sûr été décisive pour Augustin, car elle l'aura aidé, nous assure l'évêque d'Hippone, à se défaire d'une conception trop matérielle de la divinité, que le manichéisme lui avait inculquée. Aussi est-ce par la lecture des platoniciens qu'Augustin aura vécu sa première expérience de conversion, ou sa seconde après la conversion à la philosophie survenue lors de sa lecture de l'Hortensius de Cicéron - mais qui avait fini par le jeter dans les «filets» des manichéens. Les livres des platoniciens auront été un second Hortensius pour Augustin, et le conduiront enfin à la sagesse véritable. Le texte de la rencontre décisive $(7,9,13)$ est bien connu: "c'est ainsi que Tu m'as procuré [car c'est toujours Dieu qui agit dans son expérience de conversion], par l'intermédiaire d'un homme tout gonflé d'un monstrueux orgueil, certains livres des platoniciens (quosdam platonicorum libros) traduits du grec en latin». Si Augustin ne précise pas quels livres précis il a lus - sans doute s'agira-t-il de textes de Plotin et de Porphyre traduits par Marius Victorinus -, il tient cependant à dire ce qu'il y a trouvé, mais aussi ce qu'il n'y a pas trouvé:

Or j’y ai lu — non pas, bien sûr, en ces termes, mais suggéré tel, à s'y méprendre, par maintes sinueuses raisons - qu'au commencement était le Verbe, et le Verbe était en Dieu, et le Verbe était Dieu; qu'au commencement il était en Dieu; tout par lui a été fait, et sans lui rien n'a été fait; ce qui a été fait est Vie en lui, et la Vie était la Lumière des hommes; et la Lumière luit dans les ténèbres, et les ténèbres ne l'ont pas saisie, et aussi que l'âme de l'homme, tout en rendant témoignage à la Lumière n'était cependant pas elle-même Lumière, alors que le Verbe Dieu, lui, est la Lumière vraie qui illumine tout homme venant en ce monde; et aussi qu'il était dans ce monde, et le monde a été fait par lui, et le monde ne l'a pas connu'.

4. Conf. 7,9,13 (je cite ici la traduction de Cambronne). 
Augustin dit donc avoir trouvé dans les livres des platoniciens des idées qui sont très évidemment tirées du quatrième évangile. Qui plus est, ce texte de Jean dit quelque chose que les platoniciens n'auraient jamais pu accepter, à savoir que le Verbe s'est fait chair. L'idée d'incarnation leur est en effet toujours apparue sacrilège, car contraire à l'idée du divin. Augustin se donne d'ailleurs la peine de souligner qu'il n'a pu trouver cette idée d'incarnation dans leurs textes ${ }^{5}$. Mais pourquoi Augustin dit-il avoir trouvé dans les livres des platoniciens un passage qui fait écho au quatrième évangile? Même si cette pratique va à l'encontre de nos scrupules philologiques, Augustin a ici recours, comme l'a bien vu James O'Donnell', à un procédé rhétorique qui n'avait sans doute rien d'inhabituel à son époque, mais qui lui permettait de marquer l'accord de fond entre le christianisme et la philosophie la plus forte de son temps, celle du platonisme. C'était pour Augustin une manière de souligner que la sagesse chrétienne s'accordait avec la meilleure métaphysique de son époque, qui pour lui n'était pas celle de Plotin ou de Porphyre (son rapport à la métaphysique n'étant pas historique), mais celle du principe divin lui-même dont les platoniciens avaient une conception beaucoup plus fine que les manichéens.

Ce principe divin, Augustin le décrit en faisant appel à une conceptualité largement platonicienne, dont, pour l'essentiel, il ne se départira jamais. Elle insiste 1) sur son caractère immatériel; 2) sur l'idée que ce principe peut être appelé l'Être même ou l'être en soi ( «Id quod est», ou l'Idipsum, "cela même en personne ", mis en parallèle avec le texte de l'Exode où Dieu avait dit à Moïse «je suis celui qui suis ", sum qui sum), entendons l'être permanent, étranger au temps; 3) sur la possibilité (et même la réalité) d'une vision directe de cet « être même, avec l'œil de l'âme;

5. Conf. 7,9,14: "Mais que le Verbe s'est fait chair et a habité parmi nous, dans ces livres [des platoniciens], je ne l'ai pas lu. »

6. Voir O'Donnell (1992) ad 7.9.13: «Central to A.’s presentation of the doctrines of the Platonists, he employs a rhetorical device that has gone comparatively unattended. He does not quote or paraphrase the Platonic books themselves (thereby making their identification difficult), but he quotes the ipsissima verba of Christian scripture as though they offered a fair summary of contents of a non-Christian philosophical work; incidentally the device allows close comparison of the doctrines of Platonists and Christians. He has already employed the device, in his last account of reading a philosophical classic, the Hortensius (at 3.4.8, quoting Col. 2.8-9...). The syncretism he makes is one that seemed obvious at the time. " 
4) et sur l'idée, plus plotinienne que platonicienne sans doute, qu'il faut rentrer en soi-même pour découvrir cette réalité intelligible. Augustin dit, en effet, avoir retiré de ces libri platonicorum une exhortation à rentrer dans l'intime de son âme (Conf. 7,10,16: admonitus redire ad memetipsum). C'est alors, et dans un texte où l'on s'accorde à voir des emprunts au traité de Plotin «Sur le Beau » (Enn. 1,6,8), qu'Augustin dit avoir vécu sa première expérience d'extase:

avec l'œil de mon âme, quelque médiocre que fût son état, je vis, au-dessus de ce même œil de mon âme, au-dessus de mon intelligence, une lumière immuable (vidi qualicumque oculo animce mex supra eundem oculum animce mece, supra mentem meam, lucem incommutabilem), non pas cette lumière ordinaire perceptible à tout regard charnel, ni une sorte de lumière qui, tout en étant du même genre, serait plus vive, resplendirait en quelque sorte avec beaucoup, beaucoup plus de splendeur et emplirait tout de sa grandeur. Non, ce n'était pas cela qu'était cette lumière; non, elle était d'un autre, d'un tout autre ordre que tout ce monde d'ici-bas (Conf. 7,10,16; Cambronne, 917) ${ }^{7}$.

C'est là la première des deux expériences d'extase de Milan (7,10,16 et $7,17,23$ ), vécues avant la conversion (décrite au livre 8) et les extases d'Ostie du livre $9(9,10,23)$. À l'évidence, ces expériences ont été suscitées par l'enthousiasme que lui aura procuré la lecture des livres des platoniciens, qui sont bien sûr les premiers à exalter l'idée d'une vision directe de la réalité intelligible avec l'œil de l'âme (République viI, 533 d), où le platonisme aperçoit le plus grand ravissement qui puisse être atteint lors de notre existence terrestre, "là où la vie vaut la peine d'être vécue " (Banquet $211 \mathrm{~d})$.

Or, assez curieusement pour nous, Augustin insiste surtout sur ce que ces expériences d'extase avaient d'insatisfaisant. C'est pourquoi les commentateurs chrétiens, depuis Courcelle, parlent volontiers des «vaines tentatives d'extases plotiniennes» du livre 7 (Courcelle 1968, 157-167).

Si l'expression - célèbre - de Courcelle est un peu malheureuse, c'est que ces expériences n'auront pas seulement été des "tentatives", mais des visions tout à fait réelles pour Augustin. Elles n'auront pas été "vaines»

7. À rapprocher de Plotin, Ennéade 1.6 ( "Sur le Beau»), 8 et 9; 1,3,8-12. 
non plus, puisqu'elles lui auront permis — dans le cas de la deuxième extase à tout le moins $(7,17,23)^{8}$ — de se faire une idée plus juste de la divinité comme d'une réalité immuable et immatérielle. Si elles ont représenté un "échec » relatif, c'est qu'Augustin lui-même n'était pas encore assez «stable pour jouir de son Dieu " (non stabam frui deo meo): "j'étais emporté vers toi, par ta beauté, puis, bien vite, déporté loin de toi, par un poids qui m'entraînait, je m'écroulais, tout gémissant, sur ce monde d'ici-bas; et ce poids, c'était l'épaisseur de mon être de chair (pondus hoc consuetudo carnalis $)^{9}$. "Si Augustin ne pouvait se maintenir dans la vision des platoniciens, c'est qu'il était entraîné «vers le bas » par ses sinistres habitudes charnelles.

Autrement dit, un Augustin plus pur, plus «stable» aurait pu profiter plus pleinement encore de ces expériences platoniciennes. Il est vrai que dans ses réflexions sur ces expériences d'extase, Augustin insiste aussi sur les limites de la doctrine platonicienne. L'évêque d'Hippone, qui rédige ces confessions onze ans après sa conversion, les souligne volontiers et avec netteté: d'une part, le platonisme ne parlait pas de la seule voie permettant d'atteindre Dieu, celle du médiateur Jésus-Christ $(7,18,24)$; d'autre part, il n'évoquait pas non plus l'humilité essentielle à celui qui veut se revêtir du Seigneur. C'est cette leçon d'humilité qu'Augustin veut surtout retenir du mystère de l'incarnation. À la différence de ce qu'enseignent les platoniciens, Dieu n'a pas craint de s'abaisser en se faisant chair. Pour Augustin, cela veut dire que c'est aussi à un abaissement de soi que les croyants sont eux-mêmes invités. Augustin oppose volontiers cet abaissement chrétien à la présomption platonicienne qui voudrait se contenter d'une vision de la réalité intelligible, qui gonfle d'orgueil son bénéficiaire. Celle-

8. Aussi Wright $(1999,577)$ a-t-il raison de dire que la seconde ascension platonicienne (Conf. 7,17,23) peut être considérée comme réussie: «The second episode [7.17.23], later in the same book, suggests, contrary to Courcelle's assertion, a successful, if transient Plotinian ascent. Here Augustine describes himself passing through a series of stages from body to soul, from sensation to reason - that faculty "to which what is apprehended by the bodily senses is referred for judgment" - leading to reason's own self-reflection and to a perception of light and of the unchangeable. The account culminates: "Thus in a flash of its trembling sight it came to that which is. Then indeed I clearly saw your 'invisble things, understood by the things which are made'. But I was unable to fix my gaze on them”. (7.17.23) » À mes yeux, il faut aussi considérer que la première extase $(7,10,16)$ fut réussie dans la mesure où Augustin a bel et bien «vu » une lumière immuable.

9. Conf. 7,17,23 (Cambronne, 921). Voir aussi 7,20,26 (Cambronne, 925): nimis tamen infirmus ad fruendum te ("mais j'étais trop faible pour jouir de toi »). 
ci est possible, reconnaît toujours Augustin, mais pour y parvenir, le chrétien doit faire preuve de contrition et d'humble piété ${ }^{10}$. Le platonisme ferait trop appel à la présomption de l'homme, avide de contempler Dieu, et trop peu à la nécessaire et humble confession du pécheur. C'est cette humilité qui faisait encore défaut à Augustin lors de sa lecture des livres platoniciens. C'est pourquoi l'échec — tout relatif à mes yeux — des extases de Milan tient finalement autant à l'insuffisance morale d'Augustin luimême, qui se disait encore trop «infirme" (infirmus, 7,20,26) pour se maintenir dans la vision de Dieu éprouvée à Milan, qu'aux limites de la doctrine platonicienne. Le paradoxe est ici qu'Augustin souligne à la fois la vanité de cette vision et le fait qu'elle ait eu lieu! Il a bel et bien vu (vidi, conspexi) « une lumière immuable avec l'œil de son âme » (7,10,16: vidi lucem incommutabilem; 7,17,23: pervenit ad id, quod est in ictu trepidantis aspectus; tunc vero invisibilia tua per ea quo facta sunt intellecta conspexi, l'intelligence est parvenue «à ce qui est l'Être dans l'éclair d'une vision tremblante; c'est alors que je vis l'invisible de toi, qui, parmi le créé se fait voir à l'esprit "; Cambronne, 922). Il s'est donc agi d'une expérience tout à fait authentique, quelles qu'en soient les limites, qui tiennent autant, semble-t-il, à l'infirmité morale d'Augustin qu'aux insuffisances «doctrinales " du platonisme. C'est dire que le platonisme, et sa vision de la réalité essentielle (l'être même, soustrait au temps), demeure une étape importante et sans doute nécessaire dans la conversion d'Augustin.

Aussi importe-t-il de se rappeler que c'est Dieu lui-même qui a procuré à Augustin, même si ce fut par l'intermédiaire d'un homme gonflé d'orgueil (trait qui caractérise déjà le platonisme pour Augustin), ces livres des platoniciens $(7,9,13)$. Dieu en aura donc fait un moment essentiel dans la conversion d'Augustin. Le docteur de la Grâce le soulignera à la fin du livre 7:

si tu as voulu que leur rencontre précédât pour moi la méditation de tes Écritures, c'était, je pense, pour que ma mémoire gardât la marque de l'impression qu'ils m'auraient faite: une fois apprivoisé à tes livres, et une fois mes blessures pansées sous tes doigts guérisseurs, je pourrais ainsi voir et

10. Conf. 7,21,27 (Cambronne, 927): «Non, tout cela, ces livres-là ne le contiennent pas; elles ne contiennent pas, ces pages-là, le visage de cette piété, les larmes de la confession, ce sacrifice que tu aimes, l'esprit broyé, le cœur contrit d'humilité...» (non habent illo pagince vultum pietatis huius, lacrimas confessionis, sacrificium tuum, spiritum contribulatum, cor contritum et humiliatum...). 
mesurer l'écart qui sépare la présomption de la confession (discernerem atque distinguerem quid interesset inter prcesumptionem et confessionem), l'écart qui sépare ceux qui voient où il faut aller sans savoir par où, et celui qui est la Voie qui conduit non seulement à la vue, mais aussi à l'habitation de la patrie bienheureuse (inter videntes, quo eumdum sit, nec videntes qua, et viam ducentem ad beatificam patriam non tantum cernendum sed et habitandem $)^{11}$.

Si la différence entre le platonisme et le christianisme peut se résumer à celle de la présomption et de la confession, il demeure que le christianisme ne renonce nullement à l'idéal d'une vision de Dieu, dont Augustin estime qu'elle est non seulement possible, mais même essentielle ${ }^{12}$ à l'âme humaine "qui rentre en elle-même ». Nous bénéficions aussi de cette merveilleuse vision dont se targuent les platoniciens, semble dire Augustin, mais nous avons beaucoup mieux encore: la révélation directe de Dieu en la personne de son médiateur Jésus, qui s'est révélé dans ses Écritures, et qui peut conduire à une vision plus durable encore de la divinité, à savoir au repos éternel dans la maison du Seigneur. Le christianisme propose ainsi un "platonisme nouveau et amélioré », c'est-à-dire infiniment plus que la vision passagère et "tremblante" (in actu trepidantis aspectus, 7,17,23) de l'être même; il promet le repos éternel et permanent dans la maison de Dieu. Mais pour y habiter pleinement, il ne suffit pas de «croire» dans le message de salut du Verbe chrétien; il faut aussi renoncer aux tentations de la chair. Même si cet aspect doit lui permettre de dépasser le platonisme, il s'agit là aussi, assez ironiquement, d'un élément passablement "platonicien» de la conversion d'Augustin.

11. Conf. 20,26 (Cambronne, 925). Voir O'Donnell $(2005,122)$ : Augustine «falls upon books that change his life, and he lets them change his life - and then he resists them and finally leaves them behind, in favor of books that had been there whenever he wanted them all his life. Even then, what the Platonists drove him to was, he claims, a decade later, the study of Paul's letters. What he was thrilled by in the neoPlatonists was their account of mystical rapture, and Paul was the preeminent Christian authority on that subject, having been taken up to the third heaven (see 2 Corinthians 5 and 12) to see things that no one else had seen. »

12. Idée que défend excellemment Cary (2003), tout en la jugeant problématique : est-ce bien en regardant "à l'intérieur de soi » que l'on peut apercevoir la divinité et est-il juste de prétendre que cette vision soit "naturelle » à l'âme humaine ? Cary lui oppose l'idée plus thomasienne selon laquelle la vision de l'Essence divine relève non de la nature, mais de la grâce (Somme théologique. Prima pars, Q. 12, art. 4). 


\section{La scène de conversion dans le jardin de Milan: tolle et lege}

Lépisode décisif de la conversion se trouve décrit dans la scène du jardin de Milan. Je ne reviens pas ici sur les débats concernant son "historicité ", qui ont dominé les études augustiniennes depuis Courcelle. La scène paraît trop bien construite, trop inspirée par des récits comparables de conversion et des parallèles bibliques, pour être tout à fait crédible ${ }^{13}$. Notons toutefois qu'il s'agit là d'une attitude un peu moderne: lorsqu'une histoire paraît trop belle, trop construite, pour être vraie, elle est suspecte. Or, c'était le contraire qui était vrai pour Augustin: ce qui faisait l'authenticité de cette scène, c'était justement qu'elle correspondait parfaitement à des épisodes comparables de conversion. Autrement dit, notre critère d'inauthenticité ( «cela est trop beau pour être vrai») était pour Augustin, et ses contemporains, un critère d'authenticité!

Or, la question de l'authenticité n'est pas déterminante pour comprendre ce qui constitue l'essence de l'expérience qui aura été vécue sous le figuier de Milan. Augustin y entend une voix qui lui dit: "prends et lis" (tolle et lege). Il ouvre alors le texte de l'Épître aux Romains et tombe par hasard sur le verset suivant (Conf. 8,12,29): «Plus de ripailles ni de beuveries; plus de luxures ni d'impudicités; plus de disputes ni de jalousies. Revêtez-vous du Seigneur Jésus-Christ et ne vous faites pas les pourvoyeurs de la chair dans les convoitises. "Si ce texte est décisif pour Augustin, et la morale chrétienne dans son ensemble, c'est qu'il lui apprend qu'il doit renoncer une fois pour toutes à la concupiscence de la chair, qui l'empêchait de jouir pleinement de la présence divine selon le livre 7 . La conversion décisive sera donc davantage une conversion morale que doctrinale ${ }^{14}$ : Augustin s'engage alors dans une vie de continence totale, au moment précis où il lit ce texte; quand lire, c'est faire!

Le sens de cette conversion est à coup sûr celui d'une purification : ne peut recevoir le Seigneur que celui qui travaille à rendre son âme digne de

13. Voir O'Donnell $(2005,69)$ : «Reasonable skepticism can observe that the scene he describes closely resembles a gospel scene and is too well constructed to be quite believable. Augustine made no mention of any such event in the things he wrote at the time or for the ten years after until the Confessions. » Voir aussi Ferrari (2003).

14. Pour la distinction des différents aspects de la conversion d'Augustin, voir Marrou (1983, 164-165) qui distingue son côté doctrinal (l'entrée dans l'église catholique), moral (choix d'une vie d'ascèse et d'abstinence), social (Augustin renonce à sa carrière de rhéteur), philosophique (adhésion à une conception platonicienne de la divinité) et culturelle (renonciation à la culture païenne au profit d'une culture fondée sur les Écritures). 
lui. Il y a là, à n'en pas douter, un autre élément platonicien ${ }^{15}$ : Dieu ne viendra pas habiter une âme souillée. Certes, l'âme d'Augustin restait elle-même souillée, "impure ", mais Dieu n'a pu venir y habiter qu'après qu'Augustin eût renoncé aux tentations de la chair, même si cette purification aura elle-même été une opération de la grâce divine. Ainsi, la renonciation à la chair est autant celle d'Augustin que de Dieu lui-même. Son âme étant ainsi purifiée, libérée des souillures de la chair, Augustin, enfin totalement "converti », c'est-à-dire entièrement tourné, retourné, vers Dieu, se fait alors inscrire pour le baptême à la Pâque prochaine.

\section{Livre 9: Le baptême $(9,6,14-9,7,16)$ et les extases d'Ostie $(9,10,23-25)$}

On pourrait penser que le baptême d'Augustin n'aura été qu'une «formalité » pour celui qui venait de traverser une aussi renversante expérience de conversion, philosophique, religieuse et morale. Après avoir découvert la vérité de l'essence divine grâce aux livres des platoniciens et entendu la voix l'incitant à lire un texte qui exhorte lui-même à renoncer aux tentations de la chair, il ne lui restait plus qu'à se faire baptiser pour «officialiser » son adhésion à la confession chrétienne. Le baptême ne serait alors qu'une formalité cérémonielle, ce qu'il est assez largement devenu pour nous, a fortiori depuis que les spécialistes de la sacramentaire ne voient plus dans le baptême qu'un «événement communicationnel ou communautaire ». Or rien n'indique que c'est ainsi qu'Augustin ait compris les choses. Augustin a toujours reconnu au baptême, sacrement de la régénération et de la "seconde naissance ", une puissance "mystique » ou théurgique (voir O'Donnell 2005, 14 et 142), déjà évoquée lors du baptême qui a précédé la mort de son ami au livre 4.

"Nous fûmes baptisés, écrit en effet Augustin, et c'est alors que s'enfuit loin de nous l'inquiétude à propos de notre vie passée » (et baptizati sumus et fugit a nobis sollicitudo vitce prceteritce $)^{16}$. On notera aussi que cet acte du baptême sera aussitôt suivi dans les Confessions d'une "contemplation de la profondeur» (considerare altitudinem) du dessein divin: "en

15. Voir par exemple Plotin, Ennéade 1,6,9 (trad. Bréhier): «que tout être devienne donc d'abord divin et beau, s'il veut contempler Dieu et le Beau ».

16. Conf. 9,6,14 (Cambronne, 965). On sait que peu de temps après avoir rédigé ses Confessions, vers 400, Augustin a rédigé un ouvrage sur le baptême, le De baptismo contra donatistas. Inutile d'insister sur l'importance théurgique qu'Augustin reconnaissait au baptême dans son débat avec les Donatistes. 
ces jours-là, je ne me rassasiais pas de la merveilleuse douceur que je goûtais à contempler la profondeur de ton dessein sur le genre humain ${ }^{17}$ ". S'il fut une expérience mystique et théurgique, ce baptême aura préparé Augustin à une nouvelle expérience extatique, qui peut être considérée comme le terme du récit "autobiographique" des Confessions.

Si les visions de Milan firent suite à la lecture si exaltante des livres des platoniciens, la contemplation d'Ostie sera décrite, dans la trame des Confessions tout de suite après l'événement du baptême $(9,6,14-9,8,17)$ et l'hommage rendu à sa mère Monique $(9,8,17-9,9,22)$. Alors que les visions de Milan furent solitaires, la vision d'Ostie sera le fait d'une expérience partagée. Augustin l'inscrit en effet dans un dialogue qu'il a conduit avec sa mère sur la vie éternelle: «Donc, nous parlions ensemble dans un tête-à-tête fort doux. Oubliant le passé, tendus vers l'avenir, nous nous demandions entre nous, en présence de la Vérité que tu es, ce que pourrait être cette vie éternelle des saints que ni l'oeil n'a vue, ni l'oreille entendue, ni le cour de l'homme senti monter en lui [1 Co 2, 9]" (Conf. 9,10,23; Tréhorel et Bouissou, BA, t. 14, 115).

Or c'est on ne peut plus paradoxalement cette évocation d'une réalité que «ni l'œil n'a vue, ni l'oreille entendue » qui fera «monter» en Augustin et Monique l'expérience mystique d'Ostie, celle d'une élévation, degré par degré, "vers l'être même ", déjà évoqué lors de la vision de Milan $(7,17,23)$ : "nous sommes montés au-dedans de nous-même, et pendant que nous parlons et aspirons à la sagesse, voici que nous la touchons à peine, d'une poussée rapide et totale du cœur (eam attingimus modice toto ictu cordis). » "C'est alors que d'une pensée rapide nous avons atteint l'éternelle sagesse qui demeure au-dessus de tout (sicut nunc extendimus nos et rapida cogitatione attingimus aternitatem sapientiam super omnia manentem) ». Encore une fois, on pourrait mettre en question l'historicité de cette expérience $^{18}$, mais il m'apparaît significatif de souligner qu'Augustin aura voulu terminer son récit de conversion sur une expérience d'extase, qui fait très évidemment écho à celles du livre 7 (à l'in ictu trepidantis aspectus, "l'éclair d'une vision tremblante» de 7,17,23 correspond l'attingimus eam modice

17. Conf. 9,6,14 (Cambronne, 965): nec satiabar illis diebus dulcitudine mirabili, considerare altitudinem consilii tui super salutem generis humani.

18. O’Donnell souligne sans doute avec raison que, à la différence de la scène du jardin de Milan, cette expérience de la vision a suscité très peu de «littérature sceptique ". Ad locum: "The following three paragraphs are famous, too famous. And privileged: unlike the garden scene, unlike Cassiciacum, this narrative has evoked no skeptical literature debating 'the historicity of Ostia'. » 
toto ictu cordis, "d'une pensée rapide nous avons atteint l'éternelle sagesse" de 9,10,24). On pourrait dire que c'est de cette manière que sa prière d'ouverture aura été exaucée, du moins en partie: Dieu a consenti à répondre à l'appel d'Augustin, il s'est présenté à lui «en chair et en os » (ou peut-être davantage "en son", dans le cas de la vision d'Ostie). Cette expérience sera toujours décrite à l'aide d'une terminologie platonicienne, dont Augustin ne se départira jamais. Cela peut en irriter certains, ceux qui préfèrent croire au lieu de voir. Mais Augustin, lui, veut croire et voir, ou voir parce qu'il croit.

Mais, demandera-t-on, ces emprunts au platonisme ne sont-ils pas des éléments étrangers à l'expérience chrétienne de la foi ? En s'en réclamant, Augustin aura sans doute voulu signifier que l'expérience chrétienne de la foi ne le cédait en rien à l'expérience «mystique » que revendiquait la philosophie la plus avancée de son temps, celle du platonisme. Pour Augustin, la foi a beau se fonder sur des témoignages et une prédication transmise, elle est plus qu'une simple «croyance», que l'on pourrait sinon mettre sur le même pied que les autres croyances "païennes». La foi donne aussi lieu chez lui à une vision directe du divin (ou de sa «lumière»), ne laissant ainsi aucun doute sur son authenticité.

Il est vrai qu'Augustin ne voit dans cette "vision » qu'une expérience provisoire et fugitive sous les conditions de notre finitude. Les expériences d'extase ne durent qu'un instant (rapida cogitatione) et ne procurent jamais le repos ou la satisfaction d'une vision face à face. Leurs échappées de lumière ne livrent qu'un gage infime de la vision directe que promet la vie éternelle. Cela est quand même beaucoup, dira-t-on, car il s'agit là d'une «expérience mystique » à part entière, celle d'une anticipation de la vie béatifique, dont le croyant moyen d'aujourd'hui se contenterait volontiers, car elle suffirait amplement pour soutenir sa foi, voire la remplacer. Augustin voit les choses autrement. Il insiste sur le caractère passager et encore imparfait de cette extase.

Si Augustin n'y voit qu'un gage, il demeure qu'il l'intègre pleinement à l'expérience de la foi : celle-ci doit conduire à une expérience d'extase ou de vision. Or on peut se demander ici si Augustin a rendu justice à l'expérience proprement chrétienne de la foi, qui est de se savoir foi.

L' 'expérience » augustinienne d'une vision mystique de la réalité essentielle - dont il est permis de se demander, par ailleurs, si elle constitue une réelle possibilité, c'est-à-dire autre chose qu'une assurance verbale cohabite cependant avec un autre type de méditation dont les derniers livres 
des Confessions produiront la meilleure illustration, celui de la méditation de la parole divine telle qu'elle s'est exprimée dans les Écritures. À partir du livre 11 des Confessions, après les réflexions sur la mémoire et le temps, Augustin se livrera, en effet, à une patiente exégèse du texte de la Genèse. C'est elle qui nourrira durablement l'intelligence de sa foi $^{19}$, qui remplacera au livre 13 le vocabulaire de la vision mystique. Il est permis de se demander si cette méditation de la parole divine ne rend pas davantage justice à l'expérience chrétienne, car Dieu s'y donne aussi à "entendre » et peut-être plus visiblement encore que dans toute extase platonicienne.

\section{Bibliographie}

Augustin (1996 $6^{14}$ [1925]), Les Confessions, trad. P. Labriolle, Paris, Les Belles Lettres. (La Pléiade).

(1998), Les Confessions, trad. P. Cambronne, Paris, Gallimard (1962), Les Confessions, trad. E. Tréhorel et G. Bouissou, Paris, Desclée de Brouwer (Bibliothèque augustinienne, 13 et 14).

CARY, P. (2003), «Book Seven : Inner Vision as the Goal of Augustine's Life », dans K. Paffenroth et R. P. Kennedy, dir., A Reader's Companion to Augustine's Confessions, Louisville, WJK, p. 107-126.

Courcelle, P. (1968² [1950]), Recherches sur les Confessions de saint Augustin, Paris, Éditions Boccard.

FERrari, L. C. (2003), «Science and the Fictional Conversion Scene», dans K. Paffenroth et R. P. Kennedy, dir., A Reader's Companion to Augustine's Confessions, Louisville, WJK, p. 127-136.

Marrou, H.-I. (1983 [1938]), Saint Augustin et la fin de la culture antique, Paris, Éditions Boccard.

19. Voir encore O'Donnell $(2005,70)$ sur la fin du livre 9: «But the Christian story dœsn't end with death and rebirth: it holds that one must die and be reborn twice, once spiritually and once physically [...]. So, without plot, storytelling stops and a new model of existence replaces it, filling books 10 through 13 of the Confessions. Augustine the believer - the object of the narrative, seen at a distance - now has faith but not vision, believes but dœesn't really know and see, and so lives in a perpetual state of longing and a new restless alienation, loving in a new way but still imperfectly. Anxiety is joined by its sibling, hope. Wisdom [...] consists of pining for the divine, hankering after it, and struggling to get momentary glimpses and tastes of it. The language of hankering carries over from sexual love to divine love ». 
O’Donnell, J. J. (1992), Augustine: Confessions, Oxford, Clarendon Press. Collins. (2005), Augustine: A New Biography, New York, Harper

Teselle, E. (1999), "Faith», dans A. D. Fitzgerald, Augustine Through the Ages. An Encyclopedia, Grand Rapids/Cambridge, Eerdmans, p. 347-350.

Wright, R. E. (1999), "Mysticism», dans A. D. Fitzgerald, Augustine Through the Ages. An Encyclopedia, Grand Rapids/Cambridge, Eerdmans, p. 576-580.

\section{Résumé}

La figure d'Augustin est certainement exemplaire pour étudier ce dont il y va dans la relation et l'expérience religieuses. Comme chacun sait, Augustin décrit la relation religieuse comme un long processus de conversion à Dieu, dans ce qui correspond bel et bien à un acte de foi, mais cette conversion ne va pas sans certaines expériences d'extase qu'Augustin dépeint en s'inspirant de textes de Plotin. Nous nous demanderons si les deux expériences, celles de la foi et de la vision, sont compatibles, chez Augustin plus particulièrement, mais aussi sur un plan plus général.

\section{Abstract}

Augustine's work is certainly exemplary if one wishes to understand what is at stake in the religious relation and experience. It is well known that Augustine describes the religious relation as a long conversion process, a process that corresponds to an act of faith, but one that also contains elements of a direct ecstatic experience, which Augustine describes relying on the works of Plotinus. This article questions whether these two experiences, that of faith and that of vision, are compatible, in Augustine in particular but also in general. 